\title{
Workflow Automation and Portability Enable High Throughput Image Processing and Segmentation for Cell Biology Systems
}

\author{
Benjamin Provencher ${ }^{1}$, Ruslana Makovetsky ${ }^{1}$, Eric Yen $^{1}$, Nicolas Piché ${ }^{1}$, Mike Marsh ${ }^{2}$ \\ ${ }^{1 .}$ Object Research Systems. Montreal, Canada. \\ 2. Object Research Systems. Denver, USA.
}

Large scale image collection presents image analysis and annotation challenges across scientific domains, but applications in cell biology are uniquely challenging because processing routines often fail to accurately label subcellular components, leaving this task to painstaking manual processes. When advanced techniques are developed, they are often implemented as academic solutions where there is no long-term support for installation and portability to share them across the scientific community. Here we describe sharable software tools that automate image processing and segmentation (and more generic workflows), either in real-time as images are being collected or in batch for offline processing.

We build on our earlier image processing tools [1], which are TensorFlow-enabled Deep Learning tools integrated into Dragonfly, a feature-rich image processing and image visualization platform that is free for non-commercial use. These Deep Learning tools allow users to train and share neural network models that can automatically segment and denoise images with no parameter tuning. We show results on multiple biological serial section SEM experiments (both serial blockface SEM (SBFSEM) and focused ion beam SEM (FIBSEM)). Results for rat brain tissue [2] are shown in Figure 1.

For execution of routines of sequential image processing operations, we have built a macro engine that we call MacroBuilder, which allows users to record their interactive use of all features of the software, including image import, image filtering, image segmentation, etc. Besides recording their macros, users can author macros directly in our graphical macro block language. This permits users to connect operations sequentially or in loops for batch operations over multiple files.

Sometimes it is useful to process images in real-time as they are being collected. For this purpose, we developed an AutoProcess tool that monitors a folder for new images. As each image is collected, it is loaded, filtered, aligned to the stack of previous images, segmented, and rendered on screen. All of those steps are optional, and users can further customize by inserting any arbitrary macro at any step.

Deep Learning models that have been trained to accomplish a certain task can be shared with other users by uploading them to the Infinite Toolbox, an online repository for Dragonfly extensions. Likewise any of the user macros that a user runs standalone or as part of an AutoProcess recipe can be shared on Infinite Toolbox. 
The solutions described here allow users to automatically processing large datasets, harnessing the strength of Deep Learning for advanced image processing operations. The flexibility of the software enabled by the MacroBuilder lets users automate complicated workflows to fit their image pipelines.

\section{References:}

[1] R Makovetsky et al., Microscopy and Microanalysis 24 (S1) (2018), p. 532.

[2] T Eustaquio et al., Experimental Cell Research 373 (2018), p. 164.
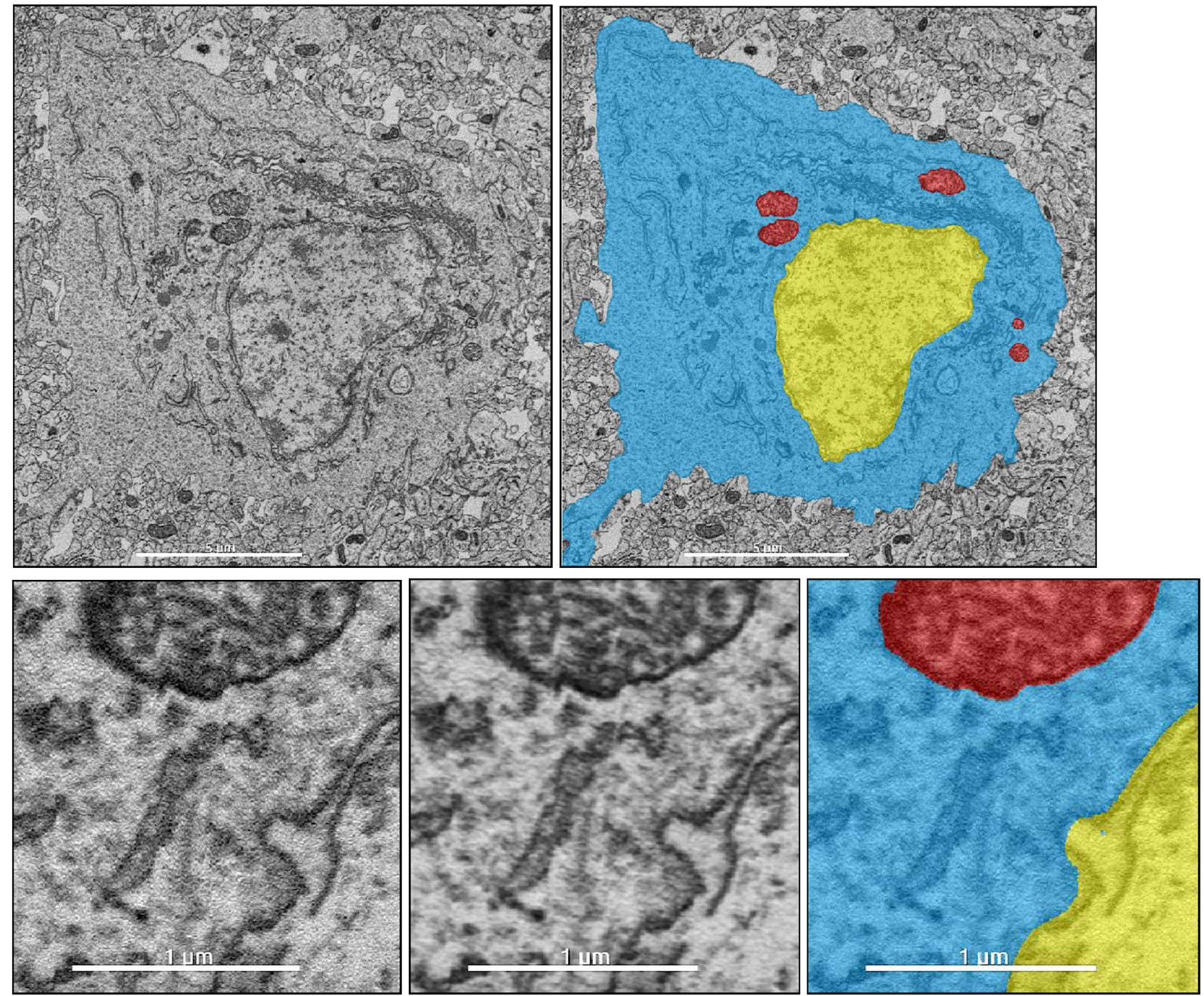

Figure 1. Fully automatic and parameter-free segmentation and image denoising of rat brain tissue. Top row: large field-of-view (scale-bar is 5um). Bottom row: zoom view (scale bar is 1um). Left: raw image. Right: automatically 4-phase segmented image. Bottom middle: automatically denoised image. Images are of rat brain tissue and were collected by SBF-SEM as described in [2]. 